\title{
PROPRIÉTÉ D'EXTENSION POUR LES COMPACTS ET RÉTRACTES ABSOLUS
}

\author{
By \\ Robert CAUTY
}

\begin{abstract}
We prove that a $\sigma$-compact metric space having the compact extension property is an AR. This solves the problem ANR 13 of West's list [5].
\end{abstract}

\section{Introduction}

Tous les espaces considérés dans cet article sont supposés métrisables et séparables

Un espace $X$ a la propriété d'extension (locale) pour les compacts si, pour tout compact $K$ et tout fermé $A$ de $K$, toute fonction continue $f: A \rightarrow X$ se prolonge en une fonction continue de $K$ (d'un voisinage de $A$ ) dans $X^{1}$. Evidemment, tout rétracte absolu (de voisinage) a la propriété d'extension (locale) pour les compacts, mais la réciproque est fausse (voir [4], [2] et le théorème 3.8 de [1]). Le problème ANR 13 de [5] demande si la réciproque est vraie lorsque $X$ est $\sigma$-compact. Le théorème suivant résout ce problème.

THÉORÈme. Soit $X$ un espace $\sigma$-compact. Si $X$ a la propriété d'extension (locale) pour les compacts, c'est un rétracte absolu (de voisinage).

La démonstration sera donnée à la section 4. La section 2 rappelle les ingrédients nécéssaires pour cette démonstration. Dans la section 3, nous montrons que tout espace ( $\sigma$-compact) ayant la propriété d'extension locale pour les compacts est un rétracte de voisinage d'un espace ( $\sigma$-compact) ayant la

2000 Mathematics Subject Classification: 54C55.

Key words and phrases: ANR, compact extension property.

${ }^{1}$ Cette propriété est équivalente à la suivante, utilisée dans la formulation du problème ANR 13 dans [5]: pour tout espace $Y$ et tout compact $A$ de $Y$, toute fonction continue $f: A \rightarrow X$ se prolonge à $Y$ (rep. à un voisinage de $A$ ) et Cette équivalence se déduit facilement de l'existence d'une fonction continue $h$ de $Y$ dans le cube de Hilbert telle que $h \mid A$ soit un plongement.

Received May 14, 2012.

Revised October 29, 2012. 
propriété d'extension pour les compacts, ce qui réduit la démonstration du théorème au cas de la propriété d'extension globale.

\section{Préliminaires}

Nous notons $I$ l'intervalle $[0,1]$.

Le résultat élémentaire suivant jouera un rôle important.

Lemme 1. Soient $Z$ un espace localement compact et $Y$ un fermé de $Z$. Si $X$ a la propriété d'extension (locale) pour les compacts, alors toute fonction continue de $Y$ dans $X$ se prolonge en une fonction continue de $Z$ (d'un voisinage de $Y$ dans Z) dans $X$.

Démonstration. Soit $g: Y \rightarrow X$ une fonction continue. Représentons $Z$ sous la forme $Z=\bigcup_{n=1}^{\infty} Z_{n}$, où $Z_{n}$ est compact et contenu dans l'intérieur de $Z_{n+1}$. Soit $Y_{n}=Y \cap Z_{n}$.

Supposons d'abord que $X$ a la propriété d'extension locale pour les compacts. Nous pouvons trouver un sous-ensemble compact $O_{1}$ de $Z_{1}$, qui est un voisinage de $Y_{1}$ dans $Z_{1}$, et une fonction continue $h_{1}: O_{1} \rightarrow X$ prolongeant $g \mid Y_{1}$. Supposons avoir construit un voisinage compact $O_{n}$ de $Y_{n}$ dans $Z_{n}$ et une fonction continue $h_{n}: O_{n} \rightarrow X$ prolongeant $g \mid Y_{n} \cup O_{n-1}\left(O_{0}=\varnothing\right)$. Définissons une fonction continue $\bar{g}_{n+1}: Y_{n+1} \cup O_{n} \rightarrow X$ par $\bar{g}_{n+1}\left|Y_{n+1}=g\right| Y_{n+1}$ et $\bar{g}_{n+1} \mid O_{n}=h_{n}$. Il existe alors un voisinage compact $O_{n+1}$ de $Y_{n+1} \cup O_{n}$ dans $Z_{n+1}$ et une fonction continue $h_{n+1}: O_{n+1} \rightarrow X$ prolongeant $\bar{g}_{n+1}$.

Soit $O=\bigcup_{n=1}^{\infty} O_{n}$. Nous définissons une fonction $h: O \rightarrow X$ en posant $h \mid O_{n}=h_{n}$ pour tout $n$. Puique $Z_{n}$ est contenu dans l'intérieur de $Z_{n+1}$ et que $O_{n+1}$ est un voisinage de $O_{n} \subset Z_{n}$ dans $Z_{n+1}$, l'intérieur de $O_{n+1}$ relativement à $Z$ contient $O_{n}$, donc $O$ est ouvert dans $Z$. Puisque les intérieurs des $O_{n}$ recouvrent $O$ et que $h \mid O_{n}=h_{n}$ est continue, $h$ est continue. Evidemment, $h$ est un prolongement de $g$ à l'ouvert $O$.

Si $X$ a la propriété d'extension pour les compacts, nous pouvons supposer que $O_{n}=Z_{n}$ pour tout $n$ dans l'argument précédent. Alors $O=Z$, et $h$ est un prolongement continu de $g$ à $Z$.

Le lemme suivant est un cas particulier d'un théorème de C. H. Dowker ([3], théorème 1)

Lemme 2. Soient $X, Y$ deux espaces métrisables, $A$ un fermé de $Y$ et $f:(Y \times\{0\}) \cup(A \times I) \rightarrow X$ une fonction continue. Si la restriction de $f \grave{a}$ 
$(Y \times\{0\}) \cup(A \times[0,1[)$ peut se prolonger continûment à un ensemble de la forme $(Y \times\{0\}) \cup V$, où $V$ est un voisinage de $A \times[0,1[$ dans $Y \times[0,1[$, alors $f$ a un prolongement continu $F: Y \times I \rightarrow X$.

\section{Cône Métrisable et Propriété D'extension}

Pour tout espace $X$, soit $C(X)=X \times I / X \times\{1\}$. Nous notons $\pi$ la projection de $X \times I$ sur $C(X), v$ le point $\pi(X \times\{1\})$, et posons $[x, t]=\pi(x, t)$. La projection de $X \times I$ sur $X$ induit une fonction $r: C(X) \backslash\{v\} \rightarrow X$, et la projection de $X \times I$ sur $I$ induit une fonction $\vartheta: C(X) \rightarrow I$. Nous définissons une topologie sur $C(X)$ en convenant que la restriction de $\pi$ à $X \times[0,1$ [ est un homéomorphisme de $X \times\left[0,1\left[\operatorname{sur} C(X) \backslash\{v\}\right.\right.$ et que les ensembles de la forme $\left.\left.\vartheta^{-1}(] \varepsilon, 1\right]\right)$ avec $0<\varepsilon<1$ forment une base de voisinages de $v$. Les fonctions $r$ et $\vartheta$ sont continues pour cette topologie. Il est facile de vérifier que $C(X)$ est régulier et à base dénombrable, donc métrisable.

Proposition. Si X a la propriété d'extension locale pour les compacts, alors $C(X)$ a la propriété d'extension pour les compacts.

Démonstration. Soient $K$ un compact, $A$ un fermé de $K$ et $f: A \rightarrow C(X)$ une fonction continue. La fonction $\alpha=\vartheta \circ f: A \rightarrow I$ est continue. Soit $\beta: K \rightarrow I$ un prolongement continu de $\alpha$. Soient $Z=\beta^{-1}\left(\left[0,1[)\right.\right.$ et $Y=Z \cap A=A \backslash f^{-1}(v)$. L'ensemble $Z$ est ouvert dans $K$, donc localement compact, et $Y$ est fermé dans $Z$. La fonction $g=r \circ f: Y \rightarrow X$ est continue. D'après le lemme 1, il existe donc un voisinage ouvert $O$ de $Y$ dans $Z$ et une fonction continue $h: O \rightarrow X$ qui prolonge $g$. Soit $\gamma: Z \rightarrow I$ une fonction continue telle que $\gamma^{-1}(0)=Y$ et $\gamma^{-1}(1)=$ $Z \backslash O$. Définissons une fonction $F: K \rightarrow C(X)$ par

$$
F(x)= \begin{cases}{[h(x), \min (1, \beta(x)+\gamma(x))]} & \text { si } x \in O \\ v & \text { si } x \notin O\end{cases}
$$

Il est clair que $F$ est continue en tout point de l'ouvert $O$. Pour prouver la continuité de $F$ en un point $x$ de $K \backslash O$, il suffit de montrer que, pour tout $\varepsilon>0$, il existe un voisinage $V$ de $x$ dans $K$ tel que $\left.\left.F(V) \subset \vartheta^{-1}(] \varepsilon, 1\right]\right)$, ce qui sera le cas si $\beta(y)+\gamma(y)>\varepsilon$ pour tout $y \in V \cap O$. Si $x \notin Z$, alors $\beta(x)=1$, et $x$ a un voisinage $V$ tel que $\beta(y)>\varepsilon$ pour tout $y \in V$, d'où $\beta(y)+\gamma(y) \geq \beta(y)>\varepsilon$ pour $y \in V \cap O$. Si $x$ appartient à $Z \backslash O$, alors $\gamma(x)=1$, et $x$ a un voisinage $V$ contenu dans $Z$ et tel que $\gamma(y)>\varepsilon$ pour tout $y \in V$, d'où $\beta(y)+\gamma(y) \geq \gamma(y)>\varepsilon$ pour $y \in V$. 
Si $x \in Y$, alors $h(x)=g(x)=r \circ f(x), \beta(x)=\alpha(x)=\vartheta \circ f(x)$ et $\gamma(x)=0$, d'où $F(x)=[r \circ f(x), \vartheta \circ f(x)]=f(x)$, et si $x \in A \backslash Y$, alors $F(x)=v=f(x)$, donc $F$ prolonge $f$.

Identifiant $X$ à $\pi(X \times\{0\}) \subset C(X), r$ devient une rétraction de l'ouvert $C(X) \backslash\{v\}$ sur $X$. Si $X$ est $\sigma$-compact, il en est de même de $X \times I$ et de $C(X)$. Nous obtenons donc le corollaire suivant.

COROLlaire. Tout espace ( $\sigma$-compact) ayant la propriété d'extension locale pour les compacts est un rétracte de voisinage d'un espace ( $\sigma$-compact) ayant la propriété d'extension pour les compacts.

\section{Démonstration Du Théorème}

Puisqu'un rétracte de voisinage d'un rétracte absolu est un rétracte absolu de voisinage, le corollaire de la dernière section entraîne qu'il suffit de démontrer le théorème dans le cas où l'espace $\sigma$-compact $X$ a la propriété d'extension pour les compacts, ce que nous supposons désormais.

Soient $Q$ le cube de Hilbert et $P=Q \times I$. Nous identifions $Q$ au sous-espace $Q \times\{0\}$ de $P$. Fixons une distance $d$ définissant la topologie de $Q$. Définissons une fonction continue $\Phi: P \times I \rightarrow P$ par

$$
\Phi((x, t), s)=(x, \max (t, s))
$$

Cette fonction vérifie $\Phi(y, 0)=y$ pour tout $y \in P$ et $\Phi(P \times] 0,1]) \subset P \backslash Q$.

Nous pouvons supposer que $X$ est un sous-ensemble de $Q$. Soit $E=$ $(P \backslash Q) \cup X$.

LEMME 3. E est un rétracte absolu.

Démonstration. Soient $Y$ un espace métrisable, $A$ un fermé de $Y$ et $f: A \rightarrow E$ une fonction continue. Puisque $P$ est un rétracte absolu, il existe une fonction continue $g: Y \rightarrow P$ telle que $g(x)=f(x)$ pour tout $x \in A$. Soit $\alpha: Y \rightarrow I$ une fonction continue telle que $\alpha^{-1}(0)=A$. Alors la fonction $F: Y \rightarrow E$ définie par

$$
F(y)=\Phi(y, \alpha(y))
$$

est un prolongement continu de $f$ à $Y$. 
Pour prouver que $X$ est un rétracte absolu, il suffit de montrer que c'est un rétracte de $E$, ce que nous ferons à l'aide du lemme suivant. Dans ce lemme, $(x, t, s)$ représentent les coordonnées d'un point de $P \times[1, \infty[=Q \times I \times$ $[1, \infty[$.

Lemme 4. Il existe un voisinage $W$ de $X \times[1, \infty[$ dans $P \times[1, \infty[$ et une fonction continue $f: W \rightarrow X$ vérifiant

a) pour tout entier $m \geq 1, d(f(x, t, s), x)<1 / m$ pour tout $(x, t, s) \in W \cap$ $(P \times[m, m+1])$.

Avant de démontrer ce lemme, montrons comment le théorème s'en déduit. Soit $V$ un voisinage fermé de $X \times[1, \infty[$ dans $E \times[1, \infty[$ contenu dans $W$. Pour $n \geq 1$, l'ensemble $O_{n}$ des points $(x, s) \in X \times[1, \infty[$ tels que $\{x\} \times[0,1 / n] \times\{s\}$ soit contenu dans l'intérieur de $V$ (relativement à $E \times[1, \infty[$ ) est ouvert dans $X \times\left[1, \infty\left[\right.\right.$, et nous avons $X \times\left[1, \infty\left[=\bigcup_{n=1}^{\infty} O_{n}\right.\right.$. Soit $\left(\lambda_{n}\right)$ une partition localement finie de l'unité sur $X \times\left[1, \infty\right.$ [ subordonnée au recouvrement ouvert $\left\{O_{n}\right\}$. Alors la fonction $\varphi: X \times[1, \infty[\rightarrow] 0,1]$ définie par

$$
\varphi(x, s)=\sum_{n=1}^{\infty} \frac{1}{n} \lambda_{n}(x, s)
$$

est continue et telle que $\{x\} \times[0, \varphi(x, s)] \times\{s\} \subset V$ pour tout $(x, s) \in X \times[1, \infty[$ puisque si $\lambda_{n}(x, 0) \neq 0$, alors $\{x\} \times[0,1 / n] \times\{s\} \subset V$. Définissons une fonction $g: X \times[1, \infty] \rightarrow X$ par

$$
g(x, s)= \begin{cases}x & \text { si } s=\infty \\ f(x, \varphi(x, s), s) & \text { si } s<\infty\end{cases}
$$

La continuité des fonctions $f$ et $\varphi$ et la condition a) entraînent que $g$ est continue. Puisque $(P \backslash Q) \times[1, \infty$ [ est localement compact, le lemme 1 nous permet de trouver une fonction continue $F:(P \backslash Q) \times[1, \infty[\rightarrow X$ telle que $F \mid V \cap((P \backslash Q) \times[1, \infty[)=f \mid V \cap((P \backslash Q) \times[1, \infty[)$. Puisque $X$ est fermé dans $E$, il existe une fonction continue $\psi: E \times[1, \infty[\rightarrow] 0,1]$ telle que $\psi \mid X \times[1, \infty[$ $=\varphi$.

Puisque $X \subset Q=Q \times\{0\} \subset P$, nous avons, par définition de $\Phi,(x, \varphi(x, s))$ $=\Phi(x, \varphi(x, s))$, donc $g(x, s)=f(\Phi(x, \varphi(x, s)), s)$ pour $x \in X$ et $s<\infty$. Pour $(x, s) \in X \times[1, \infty[$, nous avons $\psi(x, s)=\varphi(x, s)$, et le point $(\Phi(x, \psi(x, s)), s)$ appartient à $V$, d'où $F(\Phi(x, \psi(x, s)), s)=f(\Phi(x, \varphi(x, s)), s)$. 
Posons $h(x, s)=g(x, s) \quad$ si $(x, s) \in X \times[1, \infty], \quad h(y, 1)=F(\Phi(y, \psi(y, 1)), 1)$ si $y \in E$. D'après ce qui précède, ces définitions coïncident sur l'ensemble $X \times\{1\}$, et nous obtenons ainsi une fonction continue $h:(E \times\{1\}) \cup$ $(X \times[1, \infty]) \rightarrow X$.

Pour $(y, s) \in E \times[1, \infty[, \Phi(y, \psi(y, s))$ appartient à $P \backslash Q$; posant $H(y, s)=$ $F(\Phi(y, \psi(y, s)), s)$ pour $(y, s) \in E \times[1, \infty[$, nous obtenons une fonction continue $H: E \times[1, \infty[\rightarrow X$ prolongeant $h \mid(E \times\{1\}) \cup(X \times[1, \infty[)$. La fonction $h$ vérifie donc les hypothèses du lemme 2 , donc admet un prolongement continu $k: E \times[1, \infty] \rightarrow X$. Alors la fonction $r(y)=k(y, \infty)$ est une rétraction de $E$ sur $X$, d'où le théorème.

DÉmonstration du Lemme 4. Ecrivons $X=\bigcup_{n=1}^{\infty} X_{n}$, où $\left\{X_{n}\right\}$ est une suite croissante de compacts. Pour $n \geq 1$, soit $\mathscr{C}_{n}$ l'ensemble des couples $(U, \varepsilon)$ où $U$ est un voisinage ouvert de $X_{n}$ dans $Q$ et $\left.\left.\varepsilon \in\right] 0,1\right]$. Soit $\mathscr{C}=\bigcup_{n=1}^{\infty} \mathscr{C}_{n}$. Pour tout intervalle fermé borné $J$ (éventuellement réduit à un point) de $[1, \infty$ [ et tout $\eta>0$, considérons la propriété suivante, que peut ou non vérifier un élément $(U, \varepsilon)$ de $\mathscr{C}$.

$\mathscr{P}(J, \eta)$ : Si $A$ est un fermé de $\bar{U} \times[0, \varepsilon] \times J \subset P \times[1, \infty[$, et si $g: A \rightarrow X$ est une fonction continue telle que $d(g(x, t, s), x)<\varepsilon$ pour tout point $(x, t, s)$ de $A$, il existe une fonction continue $g^{\prime}: \bar{U} \times[0, \varepsilon] \times J \rightarrow X$ prolongeant $g$ et telle que $d\left(g^{\prime}(x, t, s), x\right)<\eta$ pour tout point $(x, t, s)$ de $\bar{U} \times[0, \varepsilon] \times J$.

Evidemment, si $\left(U^{\prime}, \varepsilon^{\prime}\right)$ et $(U, \varepsilon)$ sont deux éléments de $\mathscr{C}_{n}$ tels que $U^{\prime} \subset U$ et $\varepsilon^{\prime} \leq \varepsilon$ et si $(U, \varepsilon)$ a la propriété $\mathscr{P}(J, \eta)$, il en est de même de $\left(U^{\prime}, \varepsilon^{\prime}\right)$. Nous avons besoin du fait suivant.

Lemme 5. Quels que soient $n \geq 1$, l'intervalle fermé borné $J$ de [1, [ et $\eta>0$, il existe un élément $(U, \varepsilon)$ de $\mathscr{C}_{n}$ ayant la propriété $\mathscr{P}(J, \eta)$.

DÉmonstration. Supposons le contraire. Soit $\left\{U_{k}\right\}_{k=1}^{\infty}$ une suite d'ouverts de $Q$ telle que $X_{n}=\bigcap_{k=1}^{\infty} U_{k}=\bigcap_{k=1}^{\infty} \bar{U}_{k}$. Puisque $\left(U_{k}, 1 / k\right)$ n'a pas la propriété $\mathscr{P}(J, \eta)$, il existe un sous-ensemble fermé, donc compact, $A_{k}$ de $\bar{U}_{k} \times[0,1 / k] \times J$ et une fonction continue $g_{k}: A_{k} \rightarrow X$ telle que $d\left(g_{k}(x, t, s), x\right)<1 / k$ pour tout point $(x, t, s)$ de $A_{k}$, mais qu'il n'existe aucune fonction $g_{k}^{\prime}: \bar{U}_{k} \times[0,1 / k] \times J \rightarrow$ $X$ prolongeant $g_{k}$ et vérifiant $d\left(g_{k}^{\prime}(x, t, s), x\right)<\eta$ pour tout point $(x, t, s)$ de $\bar{U}_{k} \times[0,1 / k] \times J$.

Soit $S=\{0\} \cup\{1 / k \mid k \geq 1\} \subset \mathbf{R}$. Définissons des sous-ensembles $Y \subset Z$ de $S \times P \times J$ par 


$$
\begin{gathered}
Z=\left(\{0\} \times X_{n} \times J\right) \cup \bigcup_{k=1}^{\infty}\left\{\frac{1}{k}\right\} \times \bar{U}_{k} \times\left[0, \frac{1}{k}\right] \times J \\
Y=\left(\{0\} \times X_{n} \times J\right) \cup \bigcup_{k=1}^{\infty}\left\{\frac{1}{k}\right\} \times A_{k}
\end{gathered}
$$

Ces deux ensembles sont compacts. Définissons une fonction $g: Y \rightarrow X$ par $g(0, x, s)=x$ pour $(x, s) \in X_{n} \times J$ et $g(1 / k, y)=g_{k}(y)$ pour $y \in A_{k}$. L'hypothèse $d\left(g_{k}(x, t, s), x\right)<1 / k$ pour $(x, t, s) \in A_{k}$ garantit que $g$ est continue. Puisque $X$ a la propriété d'extension pour les compacts, il existe une fonction continue $g^{\prime}: Z \rightarrow X$ prolongeant $g$. Soit $\pi$ la projection de $S \times P \times J=S \times Q \times I \times J$ sur $Q$. Pour $(x, s) \in X_{n} \times J$, nous avons $g^{\prime}(0, x, s)=\pi(0, x, s)$. L'ensemble $O$ des $z \in Z$ tels que $d\left(g^{\prime}(z), \pi(z)\right)<\eta$ est un voisinage de $\{0\} \times X_{n} \times J$ dans $Z$. Puisque $\bigcap_{k=1}^{\infty} \bar{U}_{k} \times[0,1 / k] \times J=X_{n} \times J$, il existe un entier $k$ tel que $\{1 / k\} \times \bar{U}_{k} \times$ $[0,1 / k] \times J \subset O$. Définissons une fonction continue $g_{k}^{\prime}: \bar{U}_{k} \times[0,1 / k] \times J \rightarrow X$ par $g_{k}^{\prime}(z)=g^{\prime}(1 / k, z)$. Si $z$ appartient à $A_{k}$, alors $g^{\prime}(1 / k, z)=g(1 / k, z)=g_{k}(z)$, donc $g_{k}^{\prime}$ prolonge $g_{k}$. Pour $(x, t, s) \in \bar{U}_{k} \times[0,1 / k] \times J$, nous avons

$$
d\left(g_{k}^{\prime}(x, t, s), x\right)=d\left(g^{\prime}\left(\frac{1}{k}, x, t, s\right), \pi\left(\frac{1}{k}, x, t, s\right)\right)<\eta,
$$

ce qui contredit le choix de $A_{k}$ et $g_{k}$.

Pour tout entier $m \geq 1$, soit $J_{m}=[m, m+1]$. Le lemme 5 nous permet de construire inductivement un élément $\left(U_{m, 1}^{n}, \varepsilon_{m, 1}^{n}\right)$ de $\mathscr{C}_{n}$ de façon que les conditions suivantes soient vérifiées.

(1) $1 / m=\varepsilon_{m, 1}^{0}>\varepsilon_{m, 1}^{1}>\cdots>\varepsilon_{m, 1}^{n}>\cdots$.

(2) Pour $n \geq 1,\left(U_{m, 1}^{n}, \varepsilon_{m, 1}^{n}\right)$ a la propriété $\mathscr{P}\left(J_{m}, \varepsilon_{m, 1}^{n-1}\right)$.

Par récurrence sur $n$, construisons un élément $\left(U_{m, 2}^{n}, \varepsilon_{m, 2}^{n}\right)$ de $\mathscr{C}_{n}$ vérifiant

(3) $U_{m, 2}^{n} \subset U_{m, 1}^{n}$.

(4) $\varepsilon_{m, 2}^{n}<\varepsilon_{m, 1}^{n}$ et $\varepsilon_{m, 2}^{n}<\varepsilon_{m, 2}^{n-1}\left(\varepsilon_{m, 2}^{0}=1 / m\right)$.

(5) $\left(U_{m, 2}^{n}, \varepsilon_{m, 2}^{n}\right)$ a la propriété $\mathscr{P}\left(J_{m}, \min \left(\varepsilon_{m, 1}^{n}, \varepsilon_{m, 2}^{n-1}\right)\right)$.

Choisissons inductivement des éléments $\left(V_{m, 1}^{n}, \delta_{m, 1}^{n}\right)$ de $\mathscr{C}_{n}$ vérifiant les conditions suivantes (dans lesquelles on convient que $U_{0,2}^{n}=Q$ et $\varepsilon_{0,2}^{n}=1$ ).

(6) $V_{m, 1}^{n} \subset U_{m, 2}^{n} \cap U_{m-1,2}^{n}$.

(7) $\delta_{m, 1}^{n}<\min \left(\varepsilon_{m, 2}^{n}, \varepsilon_{m-1,2}^{n}\right)$ et $\delta_{m, 1}^{n}<\delta_{m, 1}^{n-1}\left(\delta_{m, 1}^{0}=1 / m\right)$.

(8) $\left(V_{m, 1}^{n}, \varepsilon_{m, 1}^{n}\right)$ a la propriété $\mathscr{P}\left(\{m\}, \min \left(\delta_{m, 1}^{n-1}, \varepsilon_{m, 2}^{n}, \varepsilon_{m-1,2}^{n}\right)\right)$. 
Choisissons enfin inductivement des éléments $\left(V_{m, 2}^{n}, \varepsilon_{m, 2}^{n}\right)$ de $\mathscr{C}_{n}$ vérifiant

(9) $V_{m, 2}^{n} \subset V_{m, 1}^{n}$.

(10) $\delta_{m, 2}^{n}<\delta_{m, 1}^{n}$ et $\delta_{m, 2}^{n}<\delta_{m, 2}^{n-1}\left(\delta_{m, 2}^{0}=1 / m\right)$.

(11) $\left(V_{m, 2}^{n}, \delta_{m, 2}^{n}\right)$ a la propriété $\mathscr{P}\left(\{m\}, \min \left(\delta_{m, 1}^{n}, \delta_{m, 2}^{n-1}\right)\right)$.

L'ensemble $V_{m}=\bigcup_{n=1}^{\infty} V_{m, 2}^{n}$ est un voisinage ouvert de $X$ dans $Q$. L'ensemble

$$
U_{m}=\left(\bigcup_{n=1}^{\infty} U_{m, 2}^{n} \times J_{m}\right) \backslash\left(\left(Q \backslash V_{m}\right) \times\{m\} \cup\left(Q \backslash V_{m+1}\right) \times\{m+1\}\right)
$$

est un voisinage ouvert de $X \times J_{m}$ dans $Q \times J_{m}$ tel que $U_{m} \cap(Q \times\{m\})=$ $V_{m} \times\{m\}$ et $U_{m} \cap(Q \times\{m+1\})=V_{m+1} \times\{m+1\}$.

Recouvrons $V_{m}$ par une famille $\mathscr{G}_{m}=\left\{G_{\alpha} \mid \alpha \in A_{m}\right\}$, localement finie dans $V_{m}$, d'ouverts de $Q$ telle que, pour tout $\alpha \in A_{m}, \bar{G}_{\alpha}$ soit contenu dans l'un des ensembles $V_{m, 2}^{n}$. Nous supposons les ensembles d'indices $A_{m}, m \geq 1$, deux à deux disjoints. Pour tout $\alpha \in A_{m}$, fixons un entier $n(\alpha)$ tel que $\bar{G}_{\alpha} \subset V_{m, 2}^{n(\alpha)}$.

A tout $\alpha \in A_{m}$, associons un nombre $\left.a_{\alpha} \in\right] m, m+1 / 2$ [, et posons $H_{\alpha}^{m}=$ [m, $a_{\alpha}\left[\right.$. Puisque la famille $\mathscr{G}_{m}$ est localement finie dans $V_{m}$, l'ensemble des indices $A_{m}$ est dénombrable, ce qui nous permet de choisir les $a_{\alpha}$ de façon que, pour tout $\eta>m$, il n'y ait qu'un nombre fini d'indices $\alpha \in A_{m}$ tels que $a_{\alpha} \geq \eta$. Cela garantit que la famille des ensembles $G_{\alpha} \times H_{\alpha}^{m}, \alpha \in A_{m}$, est localement finie dans $U_{m}$ (tout ouvert de la forme $\left.\left.Q \times\right] \eta, m+1\right]$ avec $\eta>m$ ne rencontre qu'un nombre fini de ces ensembles et, pour tout $(x, m) \in U_{m} \cap(Q \times\{m\})=V_{m}$, il y a un voisinage de $x$ dans $Q$ ne rencontrant qu'un nombre fini des $G_{\alpha}$ avec $\left.\alpha \in A_{m}\right)$. En outre, ces ensembles $G_{\alpha} \times H_{\alpha}^{m}$ sont ouverts dans $U_{m}$ et recouvrent $U_{m} \cap(Q \times\{m\})$.

A tout $\alpha \in A_{m+1}$, associons un nombre $\left.b_{\alpha} \in\right] m+1 / 2, m+1$ [, et posons $\left.\left.H_{\alpha}^{m}=\right] b_{\alpha}, m+1\right]$. Nous pouvons choisir ces nombres de façon que, pour tout $\eta<m+1$, il n'y ait qu'un nombre fini d'indices $\alpha \in A_{m+1}$ tels que $b_{\alpha} \leq \eta$, et cela garantit que la famille des ensembles $G_{\alpha} \times H_{\alpha}^{m}, \alpha \in A_{m+1}$, est localement finie dans $U_{m}$. En outre, ces ensembles $G_{\alpha} \times H_{\alpha}^{m}, \alpha \in A_{m+1}$, sont ouverts dans $U_{m}$ et recouvrent $U_{m} \cap(Q \times\{m+1\})$.

L'ensemble

$$
F_{m}=U_{m} \backslash \bigcup_{\alpha \in A_{m} \cup A_{m+1}} G_{\alpha} \times H_{\alpha}^{m}
$$

est fermé dans $U_{m}$ et disjoint de $Q \times\{m, m+1\}$. Les ensembles $U_{m, 2}^{n} \times J_{m}, n \geq 1$, sont ouverts dans $Q \times J_{m}$ et recouvrent $U_{m}$. Nous pouvons donc recouvrir le 
fermé $F_{m}$ par une famille $\left\{G_{\alpha} \times H_{\alpha}^{m} \mid \alpha \in B_{m}\right\}$ localement finie dans $U_{m}$ où, pour tout $\alpha \in B_{m}, G_{\alpha}$ est un ouvert de $Q$ tel que $\bar{G}_{\alpha}$ soit contenu dans l'un des ensembles $U_{m, 2}^{n}$ et $\left.H_{\alpha}^{m}=\right] c_{\alpha}, d_{\alpha}\left[\right.$ avec $m<c_{\alpha}<d_{\alpha}<m+1$. Nous supposons $B_{m}$ disjoint de $A_{m} \cup A_{m+1}$, et posons $\Gamma_{m}=A_{m} \cup A_{m+1} \cup B_{m}$. Pour tout $\alpha \in B_{m}$, fixons un entier $n(\alpha)$ tel que $\bar{G}_{\alpha} \subset U_{m, 2}^{n(\alpha)}$. La définition des entiers $n(\alpha)$ et les conditions (9) et (6) garantissent que $\bar{G}_{\alpha} \subset U_{m, 2}^{n(\alpha)}$ pour tout $\alpha \in \Gamma_{m}$.

Pour $\alpha \in A_{m}$, posons $\eta_{\alpha}=\delta_{m, 2}^{n(\alpha)}$, et pour $\alpha \in B_{m}$, posons $\eta_{\alpha}=\varepsilon_{m, 2}^{n(\alpha)}$. Pour $\alpha \in \Gamma_{m}$, soit $W_{\alpha}^{m}=\bar{G}_{\alpha} \times\left[0, \eta_{\alpha}\right] \times \bar{H}_{\alpha}^{m} \subset P \times J_{m}$. Posons

$$
W=\bigcup_{m=1}^{\infty} \bigcup_{\alpha \in \Gamma_{m}} W_{\alpha}^{m} .
$$

$W$ est un voisinage de $X \times[1, \infty[$ dans $P \times[1, \infty[$. En effet, soit $x \in X$. Si $m<s<m+1$, alors $(x, s)$ appartient à $U_{m}$ et il existe $\alpha \in \Gamma_{m}$ tel que $(x, s) \in$ $G_{\alpha} \times H_{\alpha}^{m}$; l'ensemble $G_{\alpha} \times\left[0, \eta_{\alpha}\right] \times H_{\alpha}^{m}$ est un voisinage de $(x, s)$ dans $P \times[1, \infty[$ contenu dans $W_{\alpha}^{m} \subset W$. Pour $m \geq 1$, il existe $\alpha \in A_{m}$ tel que $x \in G_{\alpha}$. Si $m>1$, alors $G_{\alpha} \times\left[0, \eta_{\alpha}\right] \times\left(H_{\alpha}^{m} \cup H_{\alpha}^{m-1}\right)$ est un voisinage de $(x, m)$ dans $P \times[1, \infty[$ contenu dans $W_{\alpha}^{m} \cup W_{\alpha}^{m-1} \subset W$; le cas $m=1$ est analogue.

La définition des ensembles $W_{\alpha}^{m}$ garantit que $W_{\alpha}^{m} \cap(P \times\{m\})=W_{\alpha}^{m-1} \cap$ $(P \times\{m\})$ pour tout $\alpha \in A_{m}$ si $m>1$, et que les ensembles $W_{\alpha}^{m}$ avec $\alpha \in A_{m}$ recouvrent $W \cap(P \times\{m\})$ pout tout $m$.

Ecrivons $V_{m}=\bigcup_{p=1}^{\infty} K_{m}^{p}$ où, pour tout $p, K_{m}^{p}$ est ouvert dans $Q$ et $\bar{K}_{m}^{p}$ est compact et contenu dans $K_{m}^{p+1}$. Soit $L_{m}^{p}=\bar{K}_{m}^{p} \backslash K_{m}^{p}$; convenons que $K_{m}^{0}=L_{m}^{0}=\varnothing$. Ecrivons $U_{m}=\bigcup_{q=1}^{\infty} M_{m}^{q}$ où, pour tout $q, M_{m}^{q}$ est ouvert dans $Q \times J_{m}$ et $\bar{M}_{m}^{q}$ est compact et contenu dans $M_{m}^{q+1}$. Soit $N_{m}^{q}=\bar{M}_{m}^{q} \backslash M_{m}^{q}$; convenons que $M_{m}^{0}=$ $N_{m}^{0}=\varnothing$.

La fonction $f: W \rightarrow X$ sera construite en quatre étapes.

Première étape. Pour $m, p \geq 1$, construisons la restriction de $f$ à $\hat{L}_{m}^{p}=$ $\left(L_{m}^{p} \times I \times\{m\}\right) \cap W$ de façon qu'elle vérifie la condition suivante:

$\left.\mathrm{b}_{1}\right)$ si $\quad(x, t, m) \in W_{\alpha}^{m}, \quad \alpha \in A_{m} \quad$ et $\quad n(\alpha)=n, \quad$ alors $\quad d(f(x, t, m), x)<$ $\min \left(\delta_{m, 1}^{n}, \delta_{m, 2}^{n-1}\right)$.

Pour $n \geq 1$, soit

$$
R_{m}(n)=\bigcup_{\substack{\alpha \in A_{m} \\ n(\alpha)=n}} W_{\alpha}^{m} \cap(P \times\{m\})=\bigcup_{\substack{\alpha \in A_{m} \\ n(\alpha)=n}} \bar{G}_{\alpha} \times\left[0, \eta_{\alpha}\right] \times\{m\},
$$

et soit $\hat{R}_{m}(n)=\bigcup_{j=n}^{\infty} R_{m}(j)$, de sorte que $\hat{R}_{m}(1)=W \cap(P \times\{m\})$. Par définition de $n(\alpha), \bar{G}_{\alpha}$ est contenu dans $V_{m, 2}^{n(\alpha)}$, et comme $\eta_{\alpha}=\delta_{m, 2}^{n(\alpha)}$ pour $\alpha \in A_{m}, R_{m}(n)$ est 
contenu dans $V_{m, 2}^{n} \times\left[0, \delta_{m, 2}^{n}\right] \times\{m\}$. Puisque la famille $\mathscr{G}_{m}=\left\{G_{\alpha} \mid \alpha \in A_{m}\right\}$ est localement finie dans $V_{m}$, il n'y a qu'un nombre fini d'indices $\alpha \in A_{m}$ tels que $\bar{G}_{\alpha}$ rencontre le compact $L_{m}^{p}$, donc les ensembles $\hat{L}_{m}^{p} \cap \hat{R}_{m}(n)$ sont compacts, et vides pour $n$ assez grand. Comme $\hat{R}_{m}(1) \cap \hat{L}_{m}^{p}=\hat{L}_{m}^{p}$, il suffit de construire la restriction de $f$ aux ensembles $\hat{R}_{m}(n) \cap \hat{L}_{m}^{p}$ par récurrence descendante sur $n$, en partant d'un entier $n$ assez grand pour que cette intersection soit vide. Soit $n$ un entier tel que la restriction de $f$ à $\hat{R}_{m}(n+1) \cap \hat{L}_{m}^{p}$ soit définie. Si $(x, t, m)$ est un point de $\tilde{L}_{m}^{p}(n)=R_{m}(n) \cap \hat{R}_{m}(n+1) \cap \hat{L}_{m}^{p}$, il appartient à un ensemble $W_{\alpha}^{m}$ avec $n(\alpha)=$ $n^{\prime}>n$, donc, par récurrence, d'après $\left.\mathrm{b}_{1}\right)$ et $(10), d(f(x, t, m), x)<\delta_{m, 2}^{n^{\prime}-1} \leq \delta_{m, 2}^{n}$. Puisque $\tilde{L}_{m}^{p}(n)$ est un sous-ensemble compact de $V_{m, 2}^{n} \times\left[0, \delta_{m, 2}^{n}\right] \times\{m\}$, (11) nous fournit une fonction continue $g_{n}^{2}: V_{m, 2}^{n} \times\left[0, \delta_{m, 2}^{n}\right] \times\{m\}$ prolongeant $f \mid \tilde{L}_{m}^{p}(n)$ et telle que $d\left(g_{n}^{2}(x, t, m), x\right)<\min \left(\delta_{m, 1}^{n}, \delta_{m, 2}^{n-1}\right)$. Nous prolongeons $f$ à $\hat{R}_{m}(n) \cap \hat{L}_{m}^{p}$ en posant $f(y)=g_{n}^{2}(y)$ pour $y \in R_{m}(n) \cap \hat{L}_{m}^{p}$.

Deuxième étape. Pour $m \geq 1$, construisons la restriction de $f$ à $(P \times\{m\}) \cap$ $W$ de façon qu'elle vérifie la condition suivante:

$\left.\mathrm{b}_{2}\right)$ si $\quad(x, t, m) \in W_{\alpha}^{m}, \quad \alpha \in A_{m} \quad$ et $\quad n(\alpha)=n, \quad$ alors $\quad d(f(x, t, m), x)<$ $\min \left(\delta_{m, 1}^{n-1}, \varepsilon_{m, 2}^{n}, \varepsilon_{m-1,2}^{n}\right)$.

Pour tout $\alpha \in A_{m}, \bar{G}_{\alpha}$ est contenu dans $V_{m}$, donc $W \cap(P \times\{m\})$ est contenu dans $V_{m} \times I \times\{m\} . V_{m}$ est la réunion de la suite croissante d'ouverts $\left\{K_{m}^{p}\right\}$ avec $\bar{K}_{m}^{p} \subset K_{m}^{p+1}$, donc $W \cap(P \times\{m\})$ est la réunion de la suite croissante d'ensembles relativements ouverts $\left\{W \cap\left(K_{m}^{p} \times I \times\{m\}\right)\right\}$, et il suffit de prolonger continûment $f$ à chacun des ensembles $\hat{K}_{m}^{p}=W \cap\left(\left(\bar{K}_{m}^{p} \backslash K_{m}^{p-1}\right) \times I \times\{m\}\right)$. Notons que $\hat{L}_{m}^{p^{\prime}}$ est contenu dans $\hat{K}_{m}^{p}$ si $p^{\prime}=p, p-1$, et en est disjoint si $p \neq p^{\prime} \neq p-1$.

Il n'y a qu'un nombre fini d'indices $\alpha \in A_{m}$ tels que $\bar{G}_{\alpha}$ rencontre le compact $\bar{K}_{m}^{p}$, donc les ensembles $\hat{R}_{m}(n) \cap \hat{K}_{m}^{p}$ sont compacts, et vides si $n$ est assez grand. Puisque $\hat{R}_{m}(1) \cap \hat{K}_{m}^{p}=\hat{K}_{m}^{p}$, il suffit de construire le prolongement de $f$ à $\hat{L}_{m}^{p} \cup \hat{L}_{m}^{p-1} \cup\left(\hat{R}_{m}(n) \cap \hat{K}_{m}^{p}\right)$ par récurrence descendante sur $n$, en partant d'un $n$ assez grand pour que $\hat{R}_{m}(n) \cap \hat{K}_{m}^{p}=\varnothing$.

Soit $n$ tel que la restriction de $f$ à $\hat{R}_{m}(n+1) \cap \hat{K}_{m}^{p}$ soit définie, et soit $(x, t, m) \in \tilde{K}_{m}^{p}(n)=R_{m}(n) \cap\left(\hat{L}_{m}^{p} \cup \hat{L}_{m}^{p-1} \cup\left(\hat{R}_{m}(n+1) \cap \hat{K}_{m}^{p}\right)\right)$. Il existe $\alpha \in A_{m}$ avec $n(\alpha)=n$ tel que $(x, t, m) \in W_{\alpha}^{m}$, donc si $(x, t, m)$ appartient à $\hat{L}_{m}^{p} \cup \hat{L}_{m}^{p-1}$, alors $d(f(x, t, m), x)<\delta_{m, 1}^{n}$ d'après $\left.\mathrm{b}_{1}\right) . \mathrm{Si}(x, t, m)$ appartient à $\hat{R}_{m}(n+1)$, il appartient à un ensemble $W_{\alpha^{\prime}}^{m}$ avec $n\left(\alpha^{\prime}\right)=n^{\prime}>n$, donc $d(f(x, t, m), x)<\delta_{m, 1}^{n^{\prime}-1} \leq \delta_{m, 1}^{n}$ par récurrence et (7). Puisque $R_{m}(n)$ est contenu dans $V_{m, 2}^{n} \times\left[0, \delta_{m, 2}^{n}\right] \times\{m\}$, il est aussi contenu dans $V_{m, 1}^{n} \times\left[0, \delta_{m, 1}^{n}\right] \times\{m\}$ d'après (9) et (10). Puisque $\tilde{K}_{m}^{p}$ est un sous-ensemble compact de $V_{m, 1}^{n} \times\left[0, \delta_{m, 1}^{n}\right] \times\{m\}$, (8) nous fournit une fonction continue $g_{n}^{1}: V_{m, 1}^{n} \times\left[0, \delta_{m, 1}^{n}\right] \times\{m\} \rightarrow X$ prolongeant $f \mid \tilde{K}_{m}^{p}(n)$ et telle que 
$d(f(x, t, m), x)<\min \left(\delta_{m, 1}^{n-1}, \varepsilon_{m, 2}^{n}, \varepsilon_{m-1,2}^{n}\right)$. Nous prolongeons $f$ à $\hat{R}_{m}(n)$ en posant $f(y)=g_{n}^{1}(y)$ pour $y \in R_{m}(n) \cap \hat{K}_{m}^{p}$.

Troisième étape. Notant $\tau$ l'homéomorphisme de $Q \times[1, \infty[\times I$ sur $Q \times I \times$ $[1, \infty[=P \times[1, \infty[$ défini par $\tau(x, s, t)=(x, t, s)$, construisons, pour $m, q \geq 1$, la restriction de $f$ aux ensembles $\hat{N}_{m}^{q}=W \cap \tau\left(N_{m}^{q} \times I\right)$ de façon que la condition suivante soit vérifiée:

$\left.\mathrm{b}_{3}\right)$ si $(x, t, s) \in W_{\alpha}^{m}, \alpha \in \Gamma_{m}$ et $n(\alpha)=n$, alors $d(f(x, t, s), x)<\min \left(\varepsilon_{m, 1}^{n}, \varepsilon_{m, 2}^{n-1}\right)$.

Pour $n \geq 1$, soit

$$
T_{m}(n)=\bigcup_{\substack{\alpha \in \Gamma_{m} \\ n(\alpha)=n}} W_{\alpha}^{m}=\bigcup_{\substack{\alpha \in \Gamma_{m} \\ n(\alpha)=n}} \bar{G}_{\alpha} \times\left[0, \eta_{\alpha}\right] \times \bar{H}_{\alpha} .
$$

Si $\alpha \in A_{m}$ (resp. $A_{m+1}$ ), alors $\eta_{\alpha}=\delta_{m, 2}^{n(\alpha)}$ (resp. $\left.\delta_{m+1,2}^{n(\alpha)}\right) \leq \varepsilon_{m, 2}^{n(\alpha)}$ d'après (10) et (7), et si $\alpha \in B_{m}$, alors $\eta_{\alpha}=\varepsilon_{m, 2}^{n(\alpha)}$. Puisque $\bar{G}_{\alpha}$ est contenu dans $U_{m, 2}^{n(\alpha)}$ pour tout $\alpha \in \Gamma_{m}, T_{m}(n)$ est contenu dans $U_{m, 2}^{n} \times\left[0, \varepsilon_{m, 2}^{n}\right] \times J_{m}$. Posons $\tilde{T}_{m}(n)=\bigcup_{j=n}^{\infty} T_{m}(j)$; alors $\hat{T}_{m}(1)=W \cap\left(P \times J_{m}\right)$. Puisque la famille des ensembles $\bar{G}_{\alpha} \times \bar{H}_{\alpha}$ avec $\alpha \in \Gamma_{m}$ est localement finie dans $U_{m}$, il n'y a qu'un nombre fini d'indices $\alpha \in \Gamma_{m}$ tels que $W_{\alpha}^{m} \cap \tau\left(N_{m}^{q} \times I\right) \neq \varnothing$, donc les ensembles $\hat{T}_{m}(n) \cap \hat{N}_{m}^{q}$ sont compacts, et sont vides pour $n$ assez grand. La fonction $f$ est déjà définie sur $\hat{N}_{m}^{q} \cap$ $(P \times\{m, m+1\})$, et nous construirons sa restriction aux ensembles $\hat{N}_{m}^{q} \cap\left(\hat{T}_{m}(n) \cup\right.$ $(P \times\{m, m+1\})$ par récurrence descendante sur $n$, la récurrence démarant trivialement pour $n$ assez grand.

Supposons $f$ définie sur $\hat{N}_{m}^{q} \cap \hat{T}_{m}(n+1)$. L'ensemble $\tilde{N}_{m}^{q}(n)=\hat{N}_{m}^{q} \cap T_{m}(n) \cap$ $\left(\hat{T}_{m}(n+1) \cup(P \times\{m, m+1\})\right)$ est un sous-ensemble compact de $U_{m, 2}^{n} \times\left[0, \varepsilon_{m, 2}^{n}\right]$ $\times J_{m}$. Soit $(x, t, s) \in \tilde{N}_{m}^{q}(n)$. Il existe $\alpha \in \Gamma_{m}$ tel que $n(\alpha)=n$ et que $(x, t, s) \in W_{\alpha}^{m}$. Si $s=m$ (resp. $m+1$ ), alors $\alpha \in A_{m}$ (resp. $\left.A_{m+1}\right)$ et $d(f(x, t, s), x)<\varepsilon_{m, 2}^{n}$ d'après $\left.\mathrm{b}_{2}\right)$; si $(x, t, s) \in \hat{T}_{m}(n+1)$, alors, pour un certain $n^{\prime}>n, d(f(x, t, s), x)<\varepsilon_{m, 2}^{n^{\prime}-1}$ $\leq \varepsilon_{m, 2}^{n}$ d'après (4). La condition (5) nous fournit une fonction continue $h_{n}^{2}: U_{m, 2}^{n} \times\left[0, \varepsilon_{m, 2}^{n}\right] \times J_{m} \rightarrow X$ prolongeant $f \mid \tilde{N}_{m}^{q}(n)$ et telle que $d\left(h_{n}^{2}(x, t, s), x\right)<$ $\min \left(\varepsilon_{m, 1}^{n}, \varepsilon_{m, 2}^{n-1}\right)$. Nous prolongeons $f$ à $\hat{N}_{m}^{q} \cap \hat{T}_{m}(n)$ en posant $f(y)=h_{n}^{2}(y)$ pour $y \in T_{m}(n) \cap \hat{N}_{m}^{q}$.

Quatrième étape. Pour $m \geq 1$, nous construisons la restriction de $f$ à $W \cap\left(P \times J_{m}\right)$ de façon qu'elle vérifie la condition suivante :

$\left.\mathrm{b}_{4}\right)$ si $(x, t, s) \in W_{\alpha}^{m}, \alpha \in \Gamma_{m}$ et $n(\alpha)=n$, alors $d(f(x, t, s), x)<\varepsilon_{m, 1}^{n-1}$.

Comme dans la deuxième étape, il suffit de construire la restriction de $f$ aux ensembles $\hat{M}_{m}^{q}=W \cap \tau\left(\left(\bar{M}_{m}^{q} \backslash M_{m}^{q-1}\right) \times I\right)$. Ici encore, il n'y a qu'un nombre fini d'indices $\alpha \in \Gamma_{m}$ tels que $W_{\alpha}^{m} \cap \tau\left(\bar{M}_{m}^{q} \times I\right) \neq \varnothing$, donc les ensembles $\hat{M}_{m}^{q} \cap \hat{T}_{m}(n)$ 
sont compacts et sont vides quand $n$ est assez grand. Nous construisons encore la restriction de $f$ aux ensembles $\hat{M}_{m}^{q} \cap \hat{T}_{m}(n)$ par récurrence descendante sur $n$.

Soit $n$ tel que la restriction de $f$ à $\hat{M}_{m}^{q} \cap \hat{T}_{m}(n+1)$ soit définie. La fonction $f$ est donc déjà définie sur le sous-ensemble compact

$$
\tilde{M}_{m}^{q}(n)=T_{m}(n) \cap\left(\hat{N}_{m}^{q} \cup \hat{N}_{m}^{q-1} \cup\left(\hat{M}_{m}^{q} \cap\left(\hat{T}_{m}(n+1) \cup(P \times\{m, m+1\})\right)\right)\right)
$$

de $T_{m}(n) \cap \hat{M}_{m}^{q}$. Soit $(x, t, s)$ un point de $\tilde{M}_{m}^{q}(n)$. Il existe $\alpha \in \Gamma_{m}$ tel que $n(\alpha)=n$ et $(x, t, s) \in W_{\alpha}^{m} . \mathrm{Si}(x, t, s) \in \hat{N}_{m}^{q} \cup \hat{N}_{m}^{q-1}$, alors $d(f(x, t, s), x)<\varepsilon_{m, 1}^{n}$ d'après $\left.\mathrm{b}_{3}\right)$. Si $(x, t, s) \in P \times\{m, m+1\}$, alors $d(f(x, t, s), x)<\varepsilon_{m, 2}^{n}<\varepsilon_{m, 1}^{n}$ d'après $\left.\mathrm{b}_{2}\right)$ et (4). Enfin, si $(x, t, s) \in \hat{T}_{m}(n+1)$, alors, pour un certain $n^{\prime}>n, d(f(x, t, s), x)<\varepsilon_{m, 1}^{n^{\prime}-1}$ $\leq \varepsilon_{m, 1}^{n}$ par récurrence et (1). Puisque $T_{m}(n)$ est contenu dans $U_{m, 2}^{n} \times\left[0, \varepsilon_{m, 2}^{n}\right] \times J_{m}$, il est aussi contenu dans $U_{m, 1}^{n} \times\left[0, \varepsilon_{m, 1}^{n}\right] \times J_{m}$ d'après (3) et (4). La condition (2) nous fournit une fonction continue $h_{n}^{1}: U_{m, 1}^{n} \times\left[0, \varepsilon_{m, 1}^{n}\right] \times J_{m} \rightarrow X$ prolongeant $f \mid \tilde{M}_{m}^{q}(n)$ et telle que $d\left(h_{n}^{1}(x, t, s), x\right)<\varepsilon_{m, 1}^{n-1}$. Nous prolongeons $f$ à $\hat{M}_{m}^{q} \cap \hat{T}_{m}(n)$ en posant $f(y)=h_{n}^{1}(y)$ pour $y \in T_{m}(n) \cap \hat{M}_{m}^{q}$.

Les conditions $b_{4}$ ) et (1) entraînent a).

\section{Références}

[ 1 ] J. van der Bijl, J. van Mill. Linear spaces, absolute retracts, and the compact extension property, Proc. Amer. Math. Soc. 104, 1988, 942-952.

[ 2 ] D. W. Curtis, J. van Mill. The compact extension property, General Topology and its Relations to Modern Analysis and Algebra, Proc. Sixth Prague Topological Symposium 1986 (Z. Frolik ed.), Heldermann Verlag, Berlin, 1988, 115-119.

[ 3 ] C. H. Dowker. Homotopy extension theorems, Proc. London Math. Soc. (3) 6, 1956, 100-116.

[4] J. van Mill. Another counterexample in ANR theory, Proc. Amer. Math. Soc. 97, 1986, 136138.

[5] J. E. West. Problems in infinite-dimensional topology, Open Problems in Topology (J. van Mill and G. M. Reeds, eds), Elsevier Science Publishers B. V. (North Holland), 1990, 523-597.

\section{Université Paris 6}

Institut de Mathématiques de Jussieu case 247

4 place Jussieu

75252 Paris cedex 05

E-mail: cauty@math.jussieu.fr 